studied using global OGG1 ${ }^{-/-}$mice and novel VSMC-specific transgenic mice lines expressing OGG1 or ${ }^{\mathrm{K} 338 / \mathrm{K} 341} \mathrm{OGG} 1$. BER activity was measured via incision cleavage of an 8-oxoG-containing oligonucleotide, 8-oxoG levels by ELISA, and protein binding/ interaction by immunoprecipitation and western blotting. OGG1, acetyl OGG1, p300, and 8-oxoG expression were assayed in mouse arteries and human plaques by immunohistochemistry.

Results Nuclear BER activity was defective in atherosclerotic plaque VSMCs, which correlated with a concomitant decrease in acetylation of OGG1 and OGG1 protein expression. In vitro knockout of OGG1 by CRISPR/CAS9 indicated that OGG1 is the major BER DNA glycosylase in VSMCs. Consistently, OGG1 expression increased BER activity and reduced 8-oxoG levels in vitro and in vivo, features not seen with the K338/K341 OGG1 acetylation mutant, indicating that acetylation regulates OGG1 activity in human VSMCs.

OGG1 knockout cells displayed increased sensitivity to DNA breaks induced by oxidant stress and increased apoptosis compared to control cells, measured by comet assay and Annexin-V/propidium iodide FACS staining respectively. This effect was blunted by overexpression of OGG1 but not K338/ ${ }^{\mathrm{K} 341}$ OGG1, suggesting a protective effect of OGG1 against DNA damage, and that the acetylation status of OGG1 is crucial for its function. Immunoprecipitation studies showed that p300 binds to OGG1, and this interaction was reduced under oxidative stress. Furthermore, levels of p300 were decreased in human plaque VSMCs and in response to oxidative stress, suggesting that ROS-induced regulation of OGG1 acetylation could be due to ROS-induced decreased in p300 expression and regulation of its interaction with OGG1.

Conclusions In conclusion, nuclear BER activity is reduced in human atherosclerotic plaque VSMCs, associated with reduced OGG1 acetylation and activity, potentially mediated by reduced p300. Mice with decreased and increased OGG1 will be used to study whether oxidative damage promotes plaque progression in atherosclerosis.

\section{CHANGES IN E-C COUPLING PROTEINS AND TRANSVERSE AND AXIAL TUBULAR STRUCTURES IN GUINEA PIG VENTRICULAR MUSCLE DURING PRE- AND POST-NATAL DEVELOPMENT TO ADULTHOOD}

Hanan Kashbour*, Michael Taggart, Kathryne White. Newcastle university

10.1136/heartjnl-2017-311726.164

Transverse tubules (TT), plasmalemma invaginations perpendicular to the long axis of the cardiomyocyte, facilitate rapid action potential transmission to the cell interior and efficient cardiac excitation-contraction coupling (ECC). Tubule alteration and changes in expression of proteins important for normal ECC have been noted in heart disease. The aim of this study is to explore the changes of selected ECC-related protein expressions, and transverse tubular structures, from preand post-natal cardiac development to adulthood in guinea pig ventricular muscle. Hearts were collected from guinea pigs at developmental stages: fetal (between gestation days (G) 55-68; term=G67-68); neonatal week one (NW1), two (NW2) and three (NW3); and adult. Hearts were flushed with a cold cardiologic solution and (i) left ventricles (LV) frozen for subsequent protein analysis by western blotting or (ii) retrogradely perfused with fixative and LV processed for ultrastructural examination by serial block face-scanning electron microscopy. Values are mean \pm SEM, compared by one-way ANOVA and Bonferroni posthoc test $(p<0.05)$. Expression of $\hat{\mathrm{I}}^{2}$ adrenoceptor, TT marker, increased in adults $(0.87$ \pm 0.14 fold relative to positive control) and neonates NW2 (0.90 \pm 0.04 fold) compared to fetal G55/57 (0.44 \pm 0.04 fold) or neonates NW1 $(0.5 \pm 0.04$ fold). Junctophilin2, a determinant of TT integrity, was expressed in G55/57 and, surprisingly, was invariant among the biological groupings. Analysis of digitally reconstructed (Amira 6.0) serial EM images revealed developmental changes in cardiomyocyte structure. Sarcomere length narrowed from G55/57 $(2.28 \pm 0.01 \mu \mathrm{m})$ to NW1 $(1.90 \pm 0.01 \mu \mathrm{m})$ and adult $(1.92 \pm 0.01 \mu \mathrm{m})$; assessing 250 sarcomeres. Total tubular volume increased from 0.68 $\pm 0.08 \%$ at G64/68 to $2.54 \pm 0.48 \%$ in the adult. Fetal cardiac TTs appear as early as the mid-third trimester of guinea pig pregnancy. Changes in TT/TAT abundance through pre- and post-natal development to adulthood mirror the changes in expression of some (Î́2 adrenoceptor) but not other (junctophilin2) proteins likely to be important for maturation of ECC. TT formation may start prenatally but it is less organised.

\section{LEPTIN INDUCES SCA-1+ PROGENITOR CELL MIGRATION ENHANCING NEOINTIMAL FORMATION}

Yao Xie* . King's College London

\subsection{6/heartjnl-2017-311726.165}

Background Leptin is a hormone that is predominantly secreted by white adipose tissue and thus the majority of obese individuals display high concentrations of plasma leptin. Initially believed to be solely a metabolic factor, leptin also plays a role in inflammation, vascular disease, to which Sca-1 + vascular progenitor cells within the vessel wall may contribute.

Hypothesis Leptin can influence neointimal formation by promoting the migration of Sca-1+ progenitor cells.

Methods and results $\mathrm{Sca}-1+$ progenitor cells were cultivated from the vessel wall of apoE-/- mice and purified via microbeads. The cell migration assessments included transwell and wound healing assays in vitro. The migration of Sca-1+ progenitor cells was markedly induced by leptin in a dosedependent manner $(2.420 \pm 0.222$ and $1.318 \pm 0.036)$. This migration induced by leptin was significantly inhibited by a leptin triple mutant antagonist CYT-566, ERK inhibitor or a STAT3 inhibitor. Western blot analysis revealed that leptin induced phosphorylation of STAT3 and ERK1/2, respectively, implicating the impact of these signal pathways. When applied the Sca-1+ progenitor cells from leptin receptor deficient mice for the experiments above, both the migratory ability and protein activation were markedly abolished. When endovascular injury was induced in wild-type mice by passing a 0.014 guidewire three times through the femoral artery, neointimal lesions were observed in a time-dependent manner. Immunostaining for leptin displayed an increased expression of leptin in the injured vessels. Serum ELISA for leptin assay demonstrated a peak increase at 24 hours after vessel injury. When red fluorescent protein (RFP) labelled-Sca-1+ 